\title{
BMJ Open Physician visits and medication prescriptions for major chronic diseases during the COVID-19 pandemic in Japan: retrospective cohort study
}

\author{
Itsuki Osawa (D) , ${ }^{1}$ Tadahiro Goto (D) ,,3 Yuko Asami, ${ }^{4}$ Noriharu Itoh, ${ }^{4}$ \\ Yasuyuki Kaga, ${ }^{5}$ Yuji Yamamoto, ${ }^{6}$ Yusuke Tsugawa (i) ${ }^{7,8}$
}

To cite: Osawa I, Goto T, Asami Y, et al. Physician visits and medication prescriptions for major chronic diseases during the COVID-19 pandemic in Japan: retrospective cohort study. BMJ Open 2021;11:e050938. doi:10.1136/ bmjopen-2021-050938

- Prepublication history and additional supplemental material for this paper are available online. To view these files, please visit the journal online. To view these files, please visit the journal online (http://dx.doi. org/10.1136/bmjopen-2021050938).

Received 06 March 2021 Accepted 08 July 2021

Check for updates

(C) Author(s) (or their employer(s)) 2021. Re-use permitted under CC BY-NC. No commercial re-use. See rights and permissions. Published by BMJ.

For numbered affiliations see end of article.

Correspondence to

Dr Itsuki Osawa;

ioosawa-tky@umin.ac.jp

\section{ABSTRACT}

Objectives There have been concerns that patients with chronic conditions may be avoiding in-person physician visits due to fear of COVID-19, leading to lower quality of care. We aimed to investigate changes in physician visits and medication prescriptions for chronic diseases before and during the COVID-19 pandemic at the population level. Design Retrospective cohort study.

Setting Nationwide claims data in Japan, 2018-2020. Participants Working-age population (aged 18-74 years) who visited physicians and received any prescriptions for major chronic diseases (hypertension, diabetes and dyslipidaemia) before the pandemic.

Outcome measures The outcomes were the monthly number of physician visits, the monthly proportion of physician visits and the monthly proportion of days covered by prescribed medication (PDC) during the pandemic (April-May 2020, as the first state of emergency over COVID-19 was declared on 7 April, and withdrawn nationally on 25 May).

Results Among 10346 patients who visited physicians for chronic diseases before the pandemic, we found a temporary decline in physician visits (mean number of visits was 1.9 in March vs 1.7 in April; $p<0.001$ ) and an increase in the proportion of patients who did not visit any physicians during the pandemic (15\% in March vs $24 \%$ in April; $p<0.001)$. Physician visits returned to the baseline in May (the mean number of visits: 1.8 , and the proportion of patients who did not visit any physicians: $9 \%)$. We observed no clinically meaningful difference in PDC between before and during the pandemic (eg, $87 \%$ in March vs $87 \%$ in April; $p=0.45$ ). A temporary decline in physician visits was more salient in seven prefectures with a larger number of COVID-19 cases than in other areas. Conclusions Although the number of physician visits declined right after the COVID-19 outbreak, it returned to the baseline one month later; patients were not skipping medications during the pandemic.

\section{INTRODUCTION}

The COVID-19 pandemic has dramatically changed the patterns of healthcare delivery in many countries. In the USA, the total number of in-person physician visits decreased by $30 \%-60 \%$ during the
Strengths and limitations of this study

- This is the first study that investigated the changes in physician visits and medication prescriptions for major chronic diseases before and during the COVID-19 pandemic at the population level in Japan.

- We analysed the data on working-age adults aged 18-74 years with major chronic diseases (hypertension, diabetes and dyslipidaemia) using nationwide claims data before the COVID-19 pandemic in Japan.

- The use of nationwide claims data enabled us to have large sample size and results that may be representative of the general population in Japan.

- Given our study analysed the data on working-age individuals in Japan, our study findings may not be generalisable to younger or older populations or to populations of other countries.

COVID-19 pandemic (after March 2020) compared with before the pandemic. ${ }^{1-3}$ Although the use of telemedicine has increased in the first several months of the pandemic, the decrease in the total number of in-person physician visits was larger than that could be fully offset by an increased telemedicine use in the USA. ${ }^{13}$ Similarly, in Japan, the total number of outpatient visits decreased by $20 \%$ and $25 \%$ in April and May 2020 compared with the same months in the prior year, respectively. ${ }^{45}$

Many patients with any chronic conditions, such as hypertension and diabetes mellitus, are known to be at a high risk of severe complications when infected with SARS-CoV-2, ${ }^{6-9}$ and therefore, those patients may have avoided physician visits (especially in-person visits) due to their fear of COVID-19, resulting in the deterioration of their chronic conditions. The lack of access to appropriate healthcare, such as fewer physician visits due to the COVID-19 
Table 1 Characteristics of 10346 patients with major chronic diseases

\begin{tabular}{|c|c|c|c|}
\hline Variables & $\begin{array}{l}\text { Total } \\
(n=10346)\end{array}$ & $\begin{array}{l}\text { Patients who lived in the } \\
\text { prefectures most affected } \\
\text { by COVID-19 }(n=7888)\end{array}$ & $\begin{array}{l}\text { Patients who } \\
\text { lived in the other } \\
\text { prefectures }(n=2175)\end{array}$ \\
\hline Age (year), mean (SD) & $57.5(9.6)$ & $58.1(9.5)$ & $55.4(9.4)$ \\
\hline Male gender & $6856(66)$ & $5216(66)$ & $1435(66)$ \\
\hline \multicolumn{4}{|l|}{ Area } \\
\hline $\begin{array}{l}\text { Healthcare access only in the seven prefectures most } \\
\text { affected by COVID-19 }\end{array}$ & 7888 (76) & - & - \\
\hline Healthcare access only in the other prefectures & $2175(21)$ & - & - \\
\hline $\begin{array}{l}\text { Healthcare access both in the seven prefectures most } \\
\text { affected by COVID-19 and the other prefectures }\end{array}$ & $283(3)$ & - & - \\
\hline \multicolumn{4}{|l|}{ Comorbidities } \\
\hline Hypertension & $8052(78)$ & $6121(78)$ & $1704(78)$ \\
\hline
\end{tabular}

We showed summary statistics of adult patients with major chronic conditions who visited physicians before the COVID-19 pandemic. Values represent $\mathrm{n}(\%)$, unless otherwise indicated.

outbreak, may have a substantial negative impact on their long-term management and prognoses. ${ }^{10}$ For example, a previous study showed a temporary reduction in healthcare access for patients with hypertension might be associated with a long-term increase in uncontrolled hypertension, ${ }^{11}$ which is associated with higher risks of cardiovascular events and all-cause mortality. ${ }^{12}$ In Italy, under the COVID-19 pandemic, policies such as temporarily extending prescriptions and promoting telemedicine were implemented to manage chronic diseases. ${ }^{13}$ However, evidence is limited as to the quantitative evaluation of the impact of the COVID-19 pandemic on the continuity of care and the population health consequences of the pandemic.

The Centers for Disease Control and Prevention recommend that patients with underlying medical conditions do not change their treatment plans and have at least a 30-day supply of prescribed medications on hand during the COVID-19 pandemic. ${ }^{14}$ As for medication adherence, a recent study in the USA reported that patients with asthma and Chronic obstructive pulmonary disease (COPD) had an increase in adherence to controller medications right after the declaration of the COVID-19 pandemic by the WHO in March 2020. ${ }^{15}$ However, evidence is limited as to how physician visits and medication prescriptions for patients with chronic conditions have changed during the COVID-19 pandemic at the population level.

In this context, we investigated the changes in physician visits and medication prescriptions for representative chronic diseases (ie, hypertension, type 2 diabetes mellitus (diabetes) and dyslipidaemia) before and during the COVID-19 pandemic in Japan.

\section{METHODS}

\section{Data source and study population}

We analysed data from the nationwide claims database (MinaCare database) from 1 March 2018 to 31 May 2020. The MinaCare database collects claims covering approximately $7.5 \%$ of the Japanese working-age population from large employers in 2020. This database includes both working individuals and their dependent family members within a wide range of age groups. ${ }^{16}{ }^{17}$ From this nationwide claims database, we identified 97225 adults (aged 18-74 years) who are covered with any health insurance included in the database from 1 March 2018 to 31 May 2020. Among this population, we further identified 10346 patients who visited physicians and received any prescriptions for three major chronic diseases (hypertension, diabetes and dyslipidaemia) at least once before the COVID-19 pandemic (from September 2019 to February 2020). We focused on these three diseases given the high prevalence rate. ${ }^{18} 19$ In order to identify patients with major chronic diseases (hypertension, diabetes, and dyslipidaemia), we used ICD-10 diagnostic codes and the medication therapeutic category codes in Japan (online supplemental appendix). Our definition of physician visits included all types of physician visits: in-person, online and telephone visits.

\section{Outcome measures}

The outcomes were the monthly number of physician visits, the monthly proportion of physician visits and the monthly proportion of days covered by prescribed medication (PDC) ${ }^{20}$ during the COVID-19 pandemic. The monthly PDC was calculated for each chronic disease (ie, hypertension, diabetes and dyslipidaemia) as the ratio of the number of days the patient is covered by the medication to the number of days of the month (eg, 30 days). If 


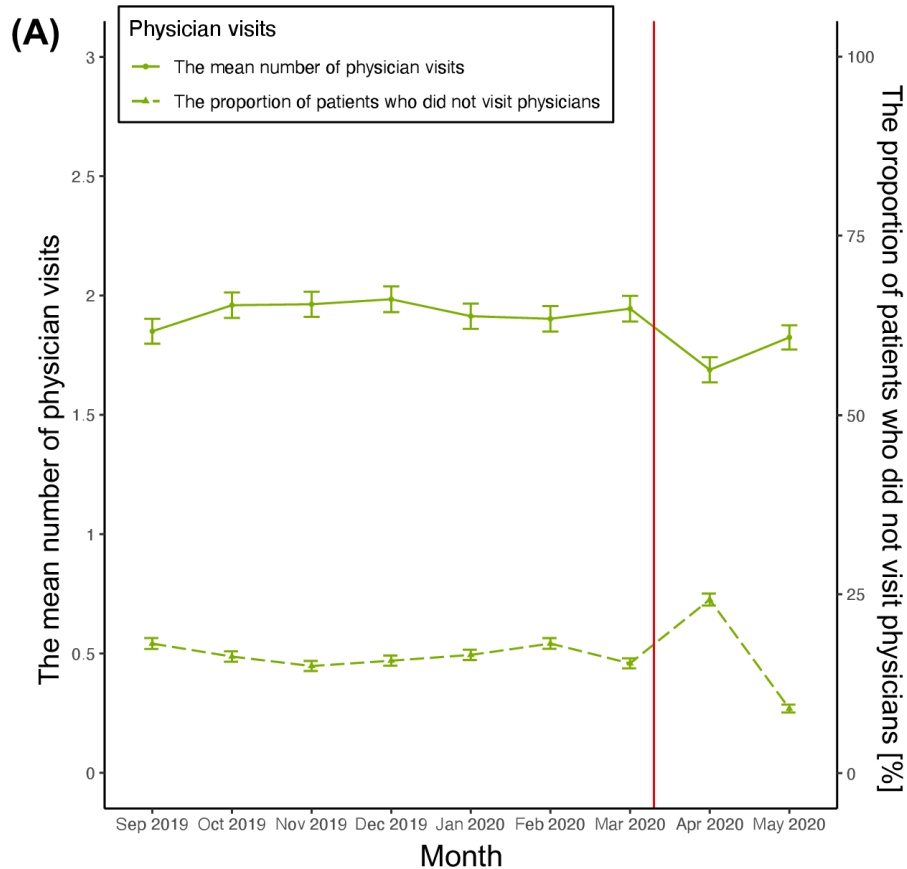

(B)

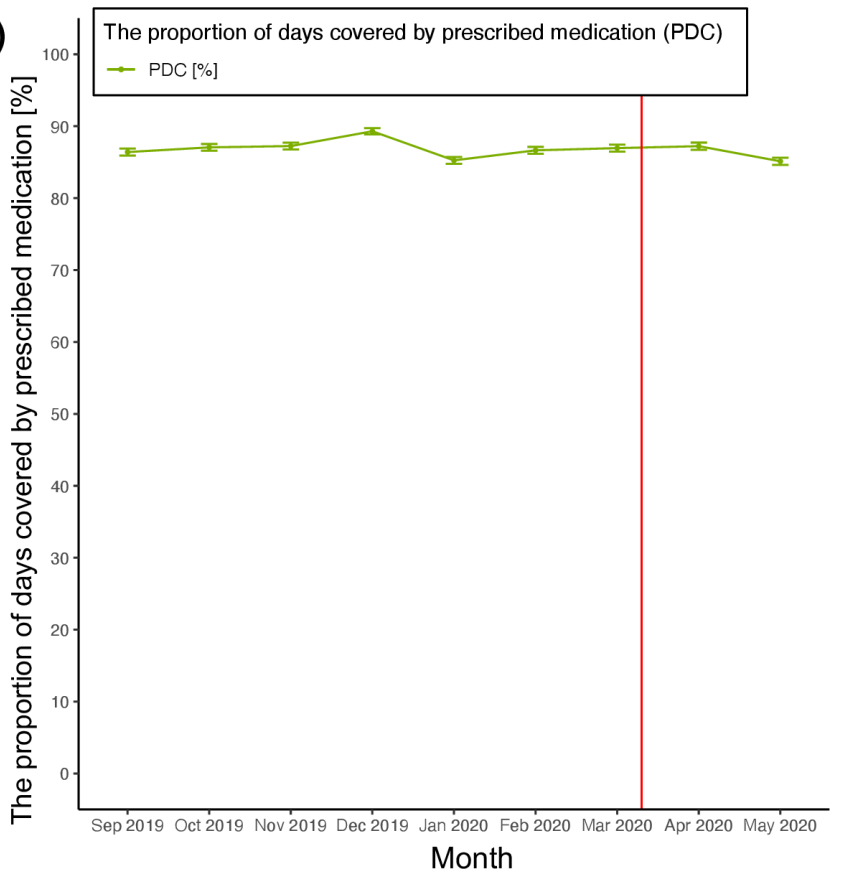

Figure 1 Changes in physician visits and medication prescriptions before and during the COVID-19 pandemic We showed the changes in physician visits and medication prescriptions in the whole nation among patients who visited physicians before the COVID-19 pandemic. The corresponding values are presented in table 2.

(A) The solid line represents the mean number of physician visits (in-person, online and telephone) per month. The dashed line represents the proportion of patients who did not visit any physicians per month. The red vertical line represents the date of the state of emergency over COVID-19.

(B) The solid line represents PDC. The red vertical line represents the date of the state of emergency over COVID- 19.

PDC, proportion of days covered by prescribed medication.

a patient had PDCs for multiple diseases (eg, hypertension and diabetes), we considered the larger value as the patient's PDC. Running out of medicine for 1 day out of a month (30 days) corresponds to a $3 \%$ reduction in PDC.

\section{Statistical analyses}

We computed summary statistics to delineate patients' characteristics. We assessed the monthly changes in the proportion of physician visits, the number of physician visits and PDC among patients who visited physicians for major chronic diseases before the COVID-19 pandemic from October 2019 to May 2020. In Japan, the state of emergency over COVID-19 was first declared in the seven most affected prefectures (Tokyo, Kanagawa, Saitama, Chiba, Osaka, Hyogo and Fukuoka) on 7 April 2020, then expanded to the national level on 16 April 2020, and finally withdrawn nationally on 25 May 2020. Therefore, we defined the COVID-19 pandemic periods as AprilMay 2020 in this study. In order to take into account non-linear changes in the use of physician visits and medication prescriptions, we analysed the data using t-test and Pearson's $\chi^{2}$ test.

\section{Secondary analyses}

We assessed the changes in outcomes stratified between patients who lived in the seven prefectures most affected by COVID-19 (Tokyo, Kanagawa, Saitama, Chiba, Osaka, Hyogo and Fukuoka) and patients who lived in the other prefectures.
In addition, to disentangle the trend during the COVID-19 pandemic from seasonal trends, we assessed the proportion of physician visits, the number of physician visits and PDC for 3 months (March-May) of 2018, 2019 and 2020 among patients with major chronic diseases who regularly visited physicians for $\geq 2$ years $(n=9036)$. The flow diagram of study participants selected for analyses is shown in online supplemental figure 1.

A $p$ value of $<0.05$ was considered statistically significant. All analyses were performed using SAS V.9.4 and R V.4.0.2. (The R Foundation for Statistical Computing) This study was a secondary data analysis of deidentified data, and therefore, the requirement for informed consent was waived.

\section{Patient and public involvement}

No patients were involved in setting the research question or the outcome measures or in developing plans for the design or implementation of the study. No patients were asked to advise on interpretation or writing up of results.

\section{RESULTS}

\section{Characteristics of the study population}

Our final sample included 10346 patients who visited physicians and received any prescriptions for representative chronic diseases before the COVID-19 pandemic. Among 10346 subjects, 6856 (66\%) were male, the mean 


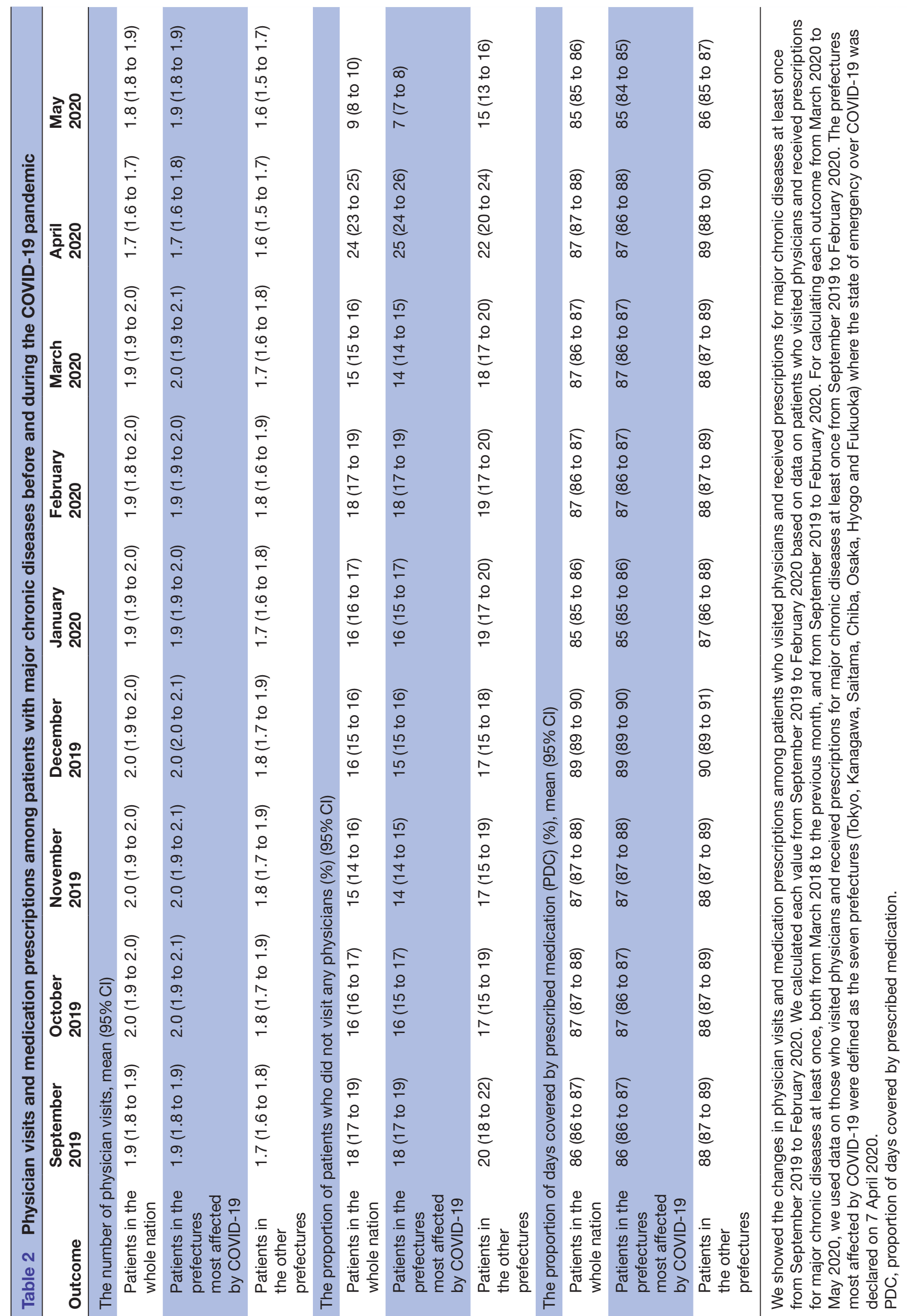



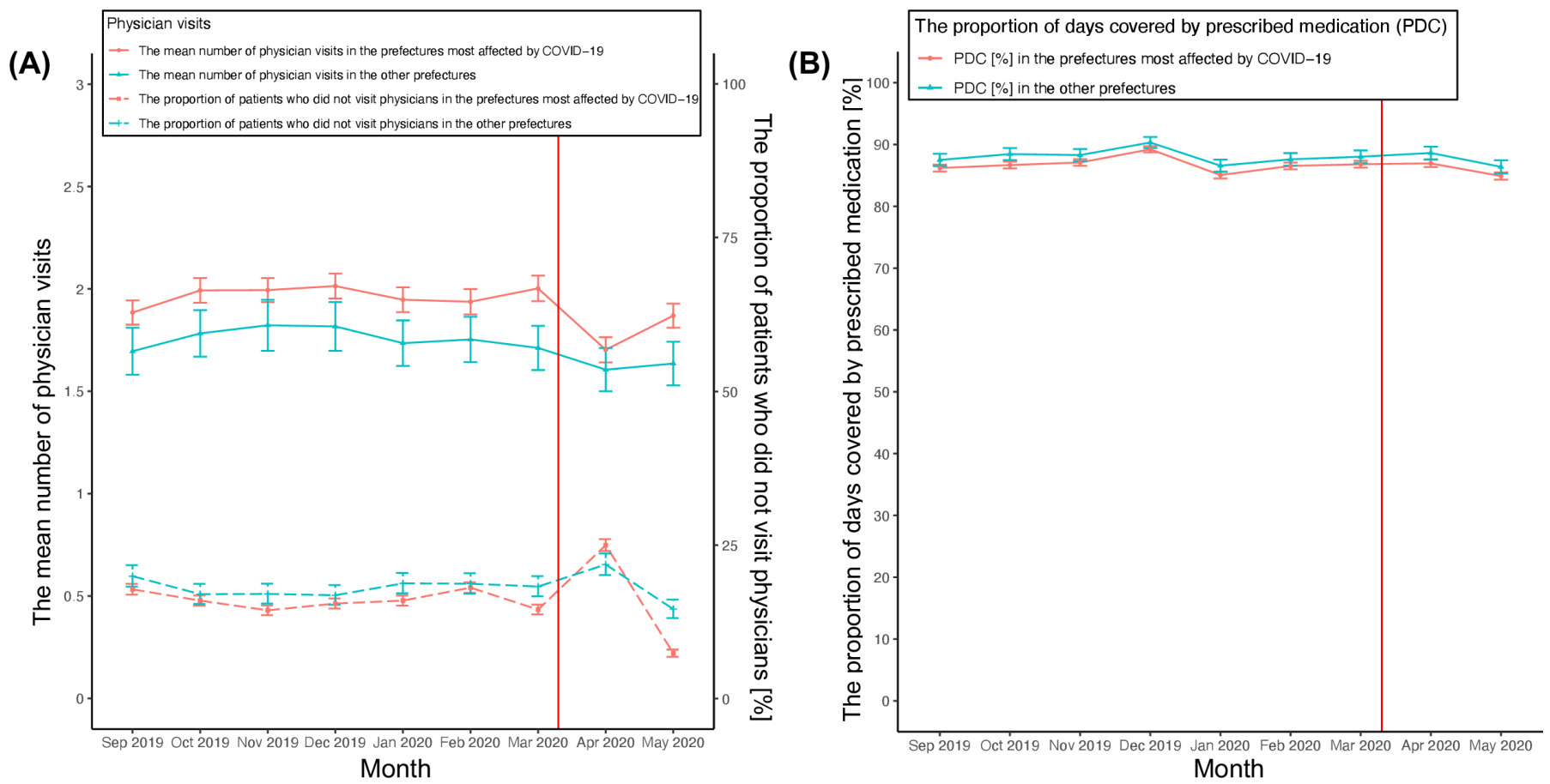

Figure 2 Changes in physician visits and medication prescriptions in the prefectures most affected by COVID-19 and the other prefectures before and during the COVID-19 pandemic

We showed the changes in physician visits and medication prescriptions in the seven prefectures most affected by COVID-19 and the other prefectures among patients who visited physicians before the COVID-19 pandemic. The corresponding values are presented in table 2.

(A) The solid line represents the mean number of physician visits (in-person, online and telephone) per month. The dashed line represents the proportion of patients who did not visit any physicians per month. The red vertical line represents the date of the state of emergency over COVID-19.

(B) The solid line represents PDC. The red vertical line represents the date of the state of emergency over COVID- 19.

PDC, proportion of days covered by prescribed medication.

(SD) age in 2018 was 58 (10) years. Of these patients, $8052(78 \%)$ had hypertension, $1532(15 \%)$ had diabetes and 4059 (39\%) had dyslipidaemia. The detailed characteristics of major chronic diseases in 10346 patients were shown in online supplemental table 1 . There were $7888(76 \%)$ patients living in the seven prefectures most affected by COVID-19 and 2175 (21\%) patients living in the other prefectures (table 1 ).

\section{Changes in physician visits and prescribed medications during the pandemic}

Figure 1 shows the monthly changes in (1) the number of physician visits, (2) the proportion of physician visits and (3) PDC among patients who visited physicians for major chronic diseases before the COVID-19 pandemic. The mean number of physician visits remained constant at around 2.0 per person-month before the COVID-19 pandemic. We found an abrupt, transient decline in the number of physician visits from 1.9 in March to 1.7 in April $(p<0.001)$, and it returned to the baseline level of 1.8 in May. The proportion of patients who did not visit any physicians remained constant at around 16\% from before the COVID-19 pandemic. We found that the number increased from $15 \%$ in March up to $24 \%$ in April $(\mathrm{p}<0.001)$, and then decreased to $9 \%$ in May. We found no evidence of changes in PDC between before and during the COVID-19 pandemic (eg, 87\% in March vs $87 \%$ in April; $\mathrm{p}=0.45$ ) (table 2).

\section{Secondary analyses}

Figure 2 shows the monthly changes in each outcome in the seven prefectures most affected by COVID-19 versus other areas among patients who visited physicians for major chronic diseases before the COVID-19 pandemic. A temporary decline in physician visits was more salient among patients living in the seven prefectures most affected by COVID-19 compared with patients living in the other prefectures. We found no difference between both areas regarding PDC-we found no changes during the pandemic in both regions (table 2).

Figure 3 shows the changes in each outcome in the whole nation from March to May in 2020 compared with the previous years (ie, 2018 and 2019) among patients who regularly visited physicians for $\geq 2$ years. In April 2020 (the beginning of the COVID-19 pandemic), there was a temporary decline in the mean number of physician visits compared with those in other study periods (eg, 1.9 in April 2018 vs 1.9 in April 2019 vs 1.7 in April 2020; $\mathrm{p}<0.001)$. Likewise, in April 2020, there was a temporary increase in the proportion of patients who did not visit any physicians compared with those in other study periods (eg, 14\% in April 2018 vs 16\% in April 2019 vs 

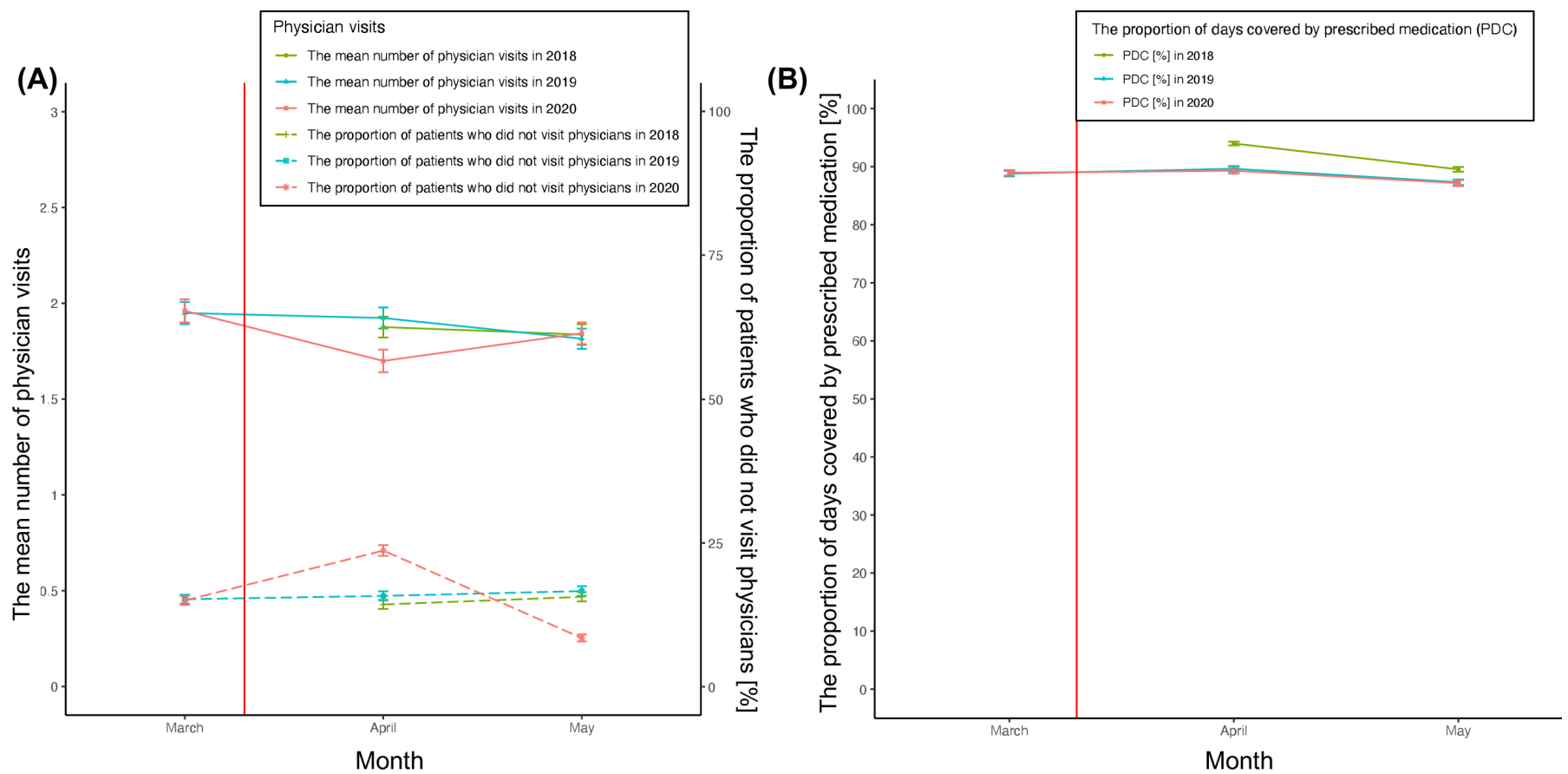

Figure 3 Changes in physician visits and medication prescriptions from March to May in 2018, 2019 and 2020

We showed the changes in physician visits and medication prescriptions in the whole nation in 2018, 2019 and 2020 among patients with major chronic diseases who visited physicians for $\geq 2$ years. Each outcome in March 2018, which could not be calculated accurately without data before February 2018, was not shown in figure 3 . The corresponding values are presented in online supplemental table 2.

(A) The solid line represents the mean number of physician visits (in-person, online and telephone) per month. The dashed line represents the proportion of patients who did not visit any physicians per month. The red vertical line represents the date of the state of emergency over COVID-19 in 2020.

(B) The solid line represents PDC. The red vertical line represents the date of the state of emergency over COVID-19 in 2020.

PDC, proportion of days covered by prescribed medication.

24\% in April 2020; $\mathrm{p}<0.001)$. However, there were no clinically meaningful changes in PDC from March to May of 2018, 2019 and 2020 (online supplemental table 2). We showed the breakdown of patients who had an in-person, online or telephone visit at least once between March and May in 2018, 2019 and 2020 in online supplemental table 3.

\section{DISCUSSION}

Using the nationwide claims database before and during the COVID-19 pandemic in Japan, we found a transient decline in the number of physician visits right after the outbreak of COVID-19, and it returned to the baseline one month later. This temporary reduction in physician visits is more salient in the seven prefectures most affected by COVID-19 than in the other prefectures. Despite these changes, we did not find any difference in PDC between before and during the COVID-19 pandemic regardless of the area status of the COVID-19 pandemic. Taken together, these findings should be reassuring for policymakers, given that they suggest that the continuity of care received by patients with chronic diseases was affected temporality due to the COVID-19 pandemic.

Our results based on patients with chronic conditions were consistent with previous papers, which have shown a decreased total number of physician visits by any patients during the COVID-19 pandemic compared with before the pandemic. ${ }^{1-5}$ Patel $e t a \hat{l}$ analysed the claims data on all types of outpatients in the USA and reported a $25 \%$ decrease in the total number of physician visits during the pandemic. This reduction was observed even though telemedicine visits became widely available as Medicare expanded reimbursement for telemedicine visits due to the COVID-19 pandemic. The current study-focusing on vulnerable patients with major chronic conditionsbuilds on these earlier studies and extends them by demonstrating a temporary reduction in physician visits even including the number of online and telephone physician visits, particularly in seven prefectures most affected by the COVID-19 pandemic. Given that essential medications for major chronic conditions have been prescribed even during the pandemic, physicians may delay their patients' next regular outpatient visits with a longer term medication prescription. In such an unprecedented situation, it is inevitable that physicians would choose a flexible approach to control patients' chronic conditions. However-as Baum et al ${ }^{11}$ reported, the 6 month closure of a healthcare system due to a hurricane increased uncontrolled hypertension, which persisted for 24 months - a transient decline in physician visits may 
have a negative impact on patients with chronic conditions in the long run.

Although we did not have any clear explanations for an increase in the number of physician visits up to the previous baseline in May 2020, this improvement could be due to the relative convergence of the COVID-19 pandemic as the state of emergency over COVID-19 was finally withdrawn nationally on 25 May 2020. Considering that the COVID-19 pandemic situation is fluctuating until the infection is well-controlled worldwide, physicians should arrange individualised schedules of regular outpatient visits and medication prescriptions based on each patient's conditions. Our findings indicate that the COVID-19 directly affected the population health through morbidity and mortality caused by the virus and had substantial indirect impacts on the chronic disease management. ${ }^{10}$ These findings shed light on the importance of the need to programme the health governance for future emergencies that account for both direct and indirect population health impact of the pandemic.

Our study has limitations. First, our data mainly included the Japanese working-age population of 18-74 years. Thus, our results may not extrapolate to the younger or older populations. Second, due to the data availability, we could not investigate the changes in physician visits and medication prescriptions for major chronic diseases over the period after May 2020. Therefore, it is possible that the patterns we observed in this study may not be different if we had a longer follow-up period and more recent data. Third, our data do not include information on patients' compliance with their prescribed medications for major chronic conditions. Lastly, since we analysed Japanese nationwide data, our findings may not be generalisable to other countries, though an abrupt decline in the number of physician visits was observed during the pandemic in the USA. ${ }^{1-3}$ Further research is needed to understand the general effect of the COVID-19 pandemic on healthcare for chronic diseases in various contexts.

\section{CONCLUSION}

The number of physician visits declined right after the surge in the number of patients with COVID-19 (April 2020); however, it returned to the baseline onemonth later (May 2020). We did not find definite evidence that patients were skipping prescribed medications for major chronic conditions during the COVID-19 pandemic.

\footnotetext{
Author affiliations

${ }^{1}$ Department of Emergency and Critical Care Medicine, The University of Tokyo Hospital, Bunkyo-ku, Tokyo, Japan

${ }^{2}$ Department of Clinical Epidemiology and Health Economics, School of Public Health, The University of Tokyo, Bunkyo-ku, Tokyo, Japan

${ }^{3}$ TXP Medical Co. Ltd, Bunkyo-ku, Tokyo, Japan

${ }^{4}$ Viatris Pharmaceuticals Japan Inc, Shibuya-ku, Tokyo, Japan

${ }^{5}$ EPS Corporation, Shinjuku-ku, Tokyo, Japan

${ }^{6}$ MinaCare Co., Ltd, Chiyoda-ku, Tokyo, Japan

${ }^{7}$ Division of General Internal Medicine and Health Service Research, David Geffen

School of Medicine at UCLA, Los Angeles, CA, USA
}

${ }^{8}$ Department of Health Policy and Management, UCLA Fielding School of Public Health, Los Angeles, CA, USA

Twitter Itsuki Osawa @0sawa_Itsuki, Tadahiro Goto @GtoDr, Yuji Yamamoto @ Yuji_Ya and Yusuke Tsugawa @ytsugawa1

Acknowledgements We would like to thank the contribution of Ms Aya Ikeda (MinaCare) for administrative support, and Ms Liying Pei and Mr Toru Yada (EPS Corporation) for the analysis of the data.

Contributors I0, TG and YT conceived and designed the study. I0, YA, NI, YK and YY had full access to all of the data in the study. All authors interpreted the results, critically revised the manuscript for important intellectual content and approved the final manuscript. YK performed the statistical analyses and is the guarantor for the integrity of the data and the accuracy of the data analysis. I0, TG and YT drafted the initial manuscript. YT supervised the study. AY, NI and YT obtained funding for this study. The corresponding author attests that all listed authors meet authorship criteria and that no others meeting the criteria have been omitted.

Funding This work was supported by Viatris Pharmaceuticals Japan Inc.

Disclaimer The content is solely the responsibility of the authors and does not necessarily represent the official views of the NIH or Viatris Pharmaceuticals Japan Inc.

Competing interests YT was supported by the National Institutes of Health (NIH)/ NIMHD Grant R01MD013913 and NIH/NIA Grant R01AG068633 for other work not related to this study. YA and NI are employees of Viatris Pharmaceuticals Japan Inc. None of the other authors were financially compensated for their effort in this project or for the development of this manuscript by Viatris Pharmaceuticals Japan Inc.

Patient consent for publication Not required.

Ethics approval The study protocol was approved by the Ethics Committee of the Research Institute of Healthcare Data Science, Tokyo, Japan.

Provenance and peer review Not commissioned; externally peer reviewed.

Data availability statement The MinaCare data are proprietary to MinaCare, Co., Ltd. and not publicly available for research purposes. Researchers who would like to access the data for research purposes should contact Dr. Yuji Yamamoto (mc info@minacare.co.jp) to make a data use agreement and pay a data-availability fee.

Supplemental material This content has been supplied by the author(s). It has not been vetted by BMJ Publishing Group Limited (BMJ) and may not have been peer-reviewed. Any opinions or recommendations discussed are solely those of the author(s) and are not endorsed by BMJ. BMJ disclaims all liability and responsibility arising from any reliance placed on the content. Where the content includes any translated material, BMJ does not warrant the accuracy and reliability of the translations (including but not limited to local regulations, clinical guidelines, terminology, drug names and drug dosages), and is not responsible for any error and/or omissions arising from translation and adaptation or otherwise.

Open access This is an open access article distributed in accordance with the Creative Commons Attribution Non Commercial (CC BY-NC 4.0) license, which permits others to distribute, remix, adapt, build upon this work non-commercially, and license their derivative works on different terms, provided the original work is properly cited, appropriate credit is given, any changes made indicated, and the use is non-commercial. See: http://creativecommons.org/licenses/by-nc/4.0/.

\section{ORCID iDs}

Itsuki Osawa http://orcid.org/0000-0001-7790-9130

Tadahiro Goto http://orcid.org/0000-0002-5880-2968

Yusuke Tsugawa http://orcid.org/0000-0002-1937-4833

\section{REFERENCES}

1 Baum A, Kaboli PJ, Schwartz MD. Reduced In-Person and increased telehealth outpatient visits during the COVID-19 pandemic. Ann Intern Med 2021;174:129-31.

2 Mehrotra A, Chernew M, Linetsky D, et al. The impact of the COVID-19 pandemic on outpatient visits: practices are adapting to the new normal. Commonwealth fund, 2021. Available: https://www. commonwealthfund.org/publications/2020/jun/impact-covid-19pandemic-outpatient-visits-practices-adapting-new-normal

3 Patel SY, Mehrotra A, Huskamp HA, et al. Trends in outpatient care delivery and telemedicine during the COVID-19 pandemic in the US. JAMA Intern Med 2021;181:388-91. 
4 Ministry of Health, Labor and Welfare. Hospital report, 2021. Available: https://www.mhlw.go.jp/toukei/saikin/hw/byouin/m19/dl/ 1905kekka.pdf

5 Ministry of Health, Labor and Welfare. Hospital report, 2021. Available: https://www.mhlw.go.jp/toukei/saikin/hw/byouin/m20/dl/ 2005kekka.pdf

$6 \mathrm{Wu}$ Z, McGoogan JM. Characteristics of and important lessons from the coronavirus disease 2019 (COVID-19) outbreak in China: summary of a report of 72314 cases from the chinese center for disease control and prevention. JAMA 2020;323:1239-42.

7 Wang D, Hu B, Hu C, et al. Clinical characteristics of 138 hospitalized patients with 2019 novel coronavirus-infected pneumonia in Wuhan, China. JAMA 2020;323:1061-9.

8 Petrilli CM, Jones SA, Yang J, et al. Factors associated with hospital admission and critical illness among 5279 people with coronavirus disease 2019 in New York City: prospective cohort study. BMJ 2020;369:m1966.

9 Williamson EJ, Walker AJ, Bhaskaran K, et al. Factors associated with COVID-19-related death using OpenSAFELY. Nature 2020;584:430-6.

10 Rosenbaum L. The untold toll - the pandemic's effects on patients without Covid-19. N Engl J Med 2020;382:2368-71.

11 Baum A, Barnett ML, Wisnivesky J, et al. Association between a temporary reduction in access to health care and long-term changes in hypertension control among Veterans after a natural disaster. JAMA Netw Open 2019;2:e1915111.

12 Pierdomenico SD, Pierdomenico AM, Coccina F, et al. Prognostic value of masked uncontrolled hypertension. Hypertension 2018;72:862-9.
13 Martini N, Piccinni C, Pedrini A, et al. CoViD-19 e malattie croniche: conoscenze attuali, passi futuri e il progetto MaCroScopio [CoViD-19 and chronic diseases: current knowledge, future steps and the MaCroScopio project. Recenti Prog Med 2020;111:198-201.

14 CDC. People with certain medical conditions, 2021. Available: https://www.cdc.gov/coronavirus/2019-ncov/need-extraprecautions/people-with-medical-conditions.html

15 Kaye L, Theye B, Smeenk I, et al. Changes in medication adherence among patients with asthma and COPD during the COVID-19 pandemic. J Allergy Clin Immunol Pract 2020;8:2384-5.

16 Shima D, li $Y$, Yamamoto $Y$, et al. A retrospective, cross-sectional study of real-world values of cardiovascular risk factors using a healthcare database in Japan. BMC Cardiovasc Disord 2014;14:120.

17 Osawa I, Goto T, Yamamoto Y, et al. Machine-learning-based prediction models for high-need high-cost patients using nationwide clinical and claims data. NPJ Digit Med 2020;3:148.

18 Saeedi P, Petersohn I, Salpea P, et al. Global and regional diabetes prevalence estimates for 2019 and projections for 2030 and 2045: results from the International Diabetes Federation Diabetes Atlas, $9^{\text {th }}$ edition. Diabetes Res Clin Pract 2019;157:107843.

19 Song Y, Liu X, Zhu X, et al. Increasing trend of diabetes combined with hypertension or hypercholesterolemia: NHANES data analysis 1999-2012. Sci Rep 2016;6:36093.

20 Choudhry NK, Shrank WH, Levin RL, et al. Measuring concurrent adherence to multiple related medications. Am J Manag Care 2009;15:457-64. 